\title{
The impact of intravenous cefazolin to the outcomes of cirrhotic patients with esophageal varices bleeding
}

\author{
Shou-Wu Lee ${ }^{1,2^{*}}$, Teng-Yu Lee ${ }^{1,2}$, Sheng-Shun Yang ${ }^{1,3}$, Chung-Wang Ko ${ }^{1,3}$, Hong-Zen Yeh ${ }^{1,3}$, \\ Chi-Sen Chang ${ }^{1,2}$ \\ ${ }^{1}$ Division of Gastroenterology and Hepatology, Department of Internal Medicine, Taichung Veterans General Hospital, Taichung, \\ Chinese Taipei \\ ${ }^{2}$ Department of Internal Medicine, Chung Shan Medical University, Taichung, Chinese Taipei \\ ${ }^{3}$ Department of Internal Medicine, Yang-Ming University School of Medicine, Taipei, Chinese Taipei \\ Email: ${ }^{*}$ ericest@vghtc.gov.tw
}

Received 11 April 2013; revised 7 May 2013; accepted 18 May 2013

Copyright (C) 2013 Shou-Wu Lee et al. This is an open access article distributed under the Creative Commons Attribution License, which permits unrestricted use, distribution, and reproduction in any medium, provided the original work is properly cited.

\begin{abstract}
Background and Method: Prophylatic antibiotics in cirrhotic patients with acute esophageal varices (EV) bleeding has been shown to increase survival and decrease the rate of bacterial infections and recurrent bleeding. This study aimed to compare the outcome of intravenous cefazolin, a first-generation cephalosporin, in the cirrhotic patients with EV bleeding. 92 consecutive cirrhotic patients with EV bleeding but without evidence of infection were retrospectively analyzed from December 2006 to February 2009, and 50 with cefazolin prophylaxis and 42 without antibiotic prophylaxis were enrolled in the antibiotic prophylaxis group and control group respectively. The outcomes were compared between both groups. Results: The incidence of infection was significantly lesser in the antibiotic prophylaxis group than that of the control group (6\% vs. $33.3 \%, P<0.05)$. The incidence of recurrent EV bleeding (6\% vs. $9.5 \%)$ and mortality (4\% vs. $7.1 \%)$ showed no significant difference between both groups. Mean length of hospital stay was shorter, but insignificant in the antibiotic prophylaxis group when compared with control group (6.6 vs. 7.8 days). Conclusion: Intravenous cefazolin can effectively reduce the rate of infection in cirrhotic patients with acute $\mathrm{EV}$ bleeding, but cannot significant benefit for preventing recurrent EV bleeding and mortality within 10 days after initial bleeding.
\end{abstract}

Keywords: Cirrhosis; Esophageal Variceal Hemorrhage; Mortality; Rebleeding

"Corresponding author.

\section{INTRODUCTION}

Patients with cirrhosis and acute esophageal variceal (EV) hemorrhage are characterized by high mortality and rebleeding rates [1]. Although rates of variceal rebleeding have been significantly reduced due to advances in endoscopic therapy, bacterial infections are frequently diagnosed in patients with cirrhosis and gastrointestinal (GI) bleeding because of their immunocompromised state, increased bacterial translocation, disruption of the intestinal mucosal barrier or frequent invasive manipulation during hemorrhage [2,3]. Previous studies have shown that bacterial infections have been documented in $22 \%$, $35 \%$ and $66 \%$ of patients within 2, 7 and 14 days after initial GI bleeding, respectively [4-6]. Control of infection of cirrhotic patients with recent bleeding decreases not only variceal rebleeding but also incidences of septic shock $[7,8]$.

Enteric bacteria are the most common agents of infection in cirrhotic patients [4]. Studies have proven the efficacy of antibiotic prophylaxis in the prevention of infection and variceal rebleeding [9-11]. The standard choices of antibiotics are oral ciprofloxacin [11], oral ofloxacin $[9,12]$, intravenous augmentin and ceftriazone [12]. The American association for the study of liver disease (AASLD) in 2007 suggested oral quinolone as a prophylactic antibiotic for cirrhotic patients with GI bleeding [13]. On the other hand, the epidemiology of bacterial infections in cirrhosis has been reported to have a higher incidence of infections caused by quinolone-resistant bacteria $[14,15]$, so ceftriaxone has been suggested as a prophylactic antibiotic in areas with a high prevalence of quinolone-resistant organisms.

However, the application of oral quinolone is usually limited due to feeding prohibition during active GI bleeding. In addition, intravenous quinolone or ceftriaxone, 
can't be used for prophylaxis due to insurance limitations in some countries. Therefore, most primary physicians in our hospital choose not to use antibiotics or intravenous first-line antibiotics, cefazoline, as prophylaxisin in clinical practice.

To the best of our knowledge, there has been no study of first-generation cephalosporins in prophylaxis for cirrhotic patients with esophageal varices (EV) bleeding. The aim of this study is to provide formal evidence for primary physicians of the effectiveness of intravenous cefazolin in the prevention of bacterial infections, early recurrent $\mathrm{EV}$ bleeding and mortality in cirrhotic patients with EV bleeding.

\section{PATIENTS AND METHODS}

Consecutive patients with liver cirrhosis and EV bleeding admitted to our hospital, a tertiary-care center, were retrospectively analyzed between December 2006 and February 2009. EV bleeding was diagnosed by UGI endoscope, and exclusion criteria included: 1) Non-cirrhotic patients, 2) EV bleeding after bacterial infections, including fever, urinary tract infection, respiratory infection and spontaneous bacterial peritonitis, 3) patients treated with antibiotics in the week before admission, 4) patients that died within 72 hours of admission, which probably meant failure to control initial EV bleeding, 5) patients that received prophylactic antibiotics other than cefazolin.

Patients were treated immediately after arriving hospital with intravenous cefazolin, 1 gm every $6-8$ hours which was adjusted according to the renal function of individuals that were placed into an antibiotic prophylaxis group, and cases without antibiotic prophylaxis that were placed into a control group. The duration of intravenous cefazolin prescribed in most patients in the antibiotic prophlyaxis group was 5 to 7 days after initial EV bleeding.

The medical records including vital signs, number of units of blood transfusion, bacterial cultures and blood leukocyte counts of patients were reviewed throughout hospitalization. Liver cirrhosis was diagnosed by medical history, physical findings, laboratory tests and radiology. The definition of infection was made by evidence of infection, such as bacteremia, urinary tract infection (UTI), respiratory infection or spontaneous bacterial peritonitis (SBP) within 10 days of admission. The diagnosis of bacteremia was made by positive results of blood cultures after initial EV bleeding and a clinical picture consistent with this diagnosis. UTI was diagnosed with pyuria and a positive culture of urine. Respiratory infection was diagnosed using clinical, radiological and bacteriological data. The diagnosis of SBP was made by positive cultures of ascites or a white cell count greater than 500 neutrophils/ $\mu \mathrm{L}$ with neutrophils greater than $50 \%$ in ascites.
The definition of EV severity grading F1, F2 and F3 was defined by mean of small straight varices, enlarged tortuous varices less than one-third of the lumen and large coil-shaped varices more than one-third of the lumen, respectively. The application of different vasoactive medication, such as sandostatin or glypressin, depended on decisions of primary physicians. No beta-blocker was given to any patient in either group during the admission period, and all patients underwent EV ligations in the initial endoscopy.

To assess the efficacy of cefazolin, the incidence of infections, ratio of recurrent $\mathrm{EV}$ bleeding, mortality rate within 10 days after admission and length of hospital stay were compared between the two groups. Patients with evidence of infections during admission period were added or shifted to proper antibiotics according to microorganisms cultured or clinical pictures as necessary.

Data was expressed as standard derivation of mean for each of the measured parameters. A P-value below 0.05 was considered statistically significant. Statistical comparisons were made between the antibiotic prophylaxis group and the control group using Mann-Whitney $U$ test to compare white blood cell count, albumin, prothombin time, creatinine, blood pressure and number of units of blood transfusion; Pearson chi-square test was used for Child-Pugh classification, severity grading of EV, etiology of cirrhosis and choice of vasoactive medication; Yate's correction of contingency for infection rate and number of EV ligation; Fisher's exact test to mortality within 10 days after admission and recurrent $\mathrm{EV}$ bleeding for categorical variables.

\section{RESULTS}

All 125 consecutive patients with cirrhosis and EV bleeding were collected, and 33 cases were excluded ( 15 patients suffered from infection on admission, 5 patients accepted antibiotics 3 days before admission, 1 patient died due to failure to control initial EV bleeding on the second hospital day, 12 patients accepted prophylactic antibiotics other than cafazolin, including ceftriaxone, quinolone, amongst others). Finally, 92 patients were included in this study. Among these cases, 50 patients who received intravenous cefazolin for prophylaxis were enrolled in the antibiotic prophylaxis group, while the 42 patients who did not receive prophylactic antibiotics were enrolled in the control group.

Clinical and laboratory characteristics of patients upon admission are displayed in Table $\mathbf{1}$, and no significant difference existed between age, gender, systolic blood pressure, number of units of blood transfusion, ascites, etiology of cirrhosis, Child-Pugh classification, severity grading of EV, white blood cell count, albumin, prothombin time and creatinin for control group and antibiotic prophylaxis group. 
Table 1. Clinical and laboratory characteristics on admissions.

\begin{tabular}{|c|c|c|c|c|c|c|}
\hline \multirow{2}{*}{\multicolumn{2}{|c|}{ Variable }} & \multicolumn{2}{|c|}{ Control Group $(n=42)$} & \multicolumn{2}{|c|}{ Antibiotic Prophylaxis Group $(\mathrm{n}=50)$} & \multirow{2}{*}{ P-value } \\
\hline & & $\mathrm{n}(\%)$ & $\mathrm{M} \pm \mathrm{SD}$ & $\mathrm{n}(\%)$ & $\mathrm{M} \pm \mathrm{SD}$ & \\
\hline \multicolumn{2}{|c|}{ Mean age (years-old) } & & $57.26 \pm 13.96$ & & $54.46 \pm 10.66$ & 0.468 \\
\hline \multicolumn{2}{|c|}{ Gender (male) } & $35(83.3 \%)$ & & $38(76.0 \%)$ & & 0.544 \\
\hline \multicolumn{2}{|c|}{ SBP (mmHg) } & & $101.46 \pm 15.01$ & & $95.60 \pm 11.76$ & 0.138 \\
\hline \multicolumn{2}{|c|}{ Blood transfusion (unit) } & & $3.89 \pm 4.67$ & & $4.73 \pm 4.66$ & 0.172 \\
\hline \multicolumn{2}{|c|}{ White blood cell count $(\times / \mu \mathrm{L})$} & & $9207.14 \pm 5239.27$ & & $8297.00 \pm 4046.87$ & 0.447 \\
\hline \multicolumn{2}{|c|}{ Albumin (g/dL) } & & $2.94 \pm 0.62$ & & $2.95 \pm 0.49$ & 0.997 \\
\hline \multicolumn{2}{|c|}{ Prothombin time (seconds) } & & $13.06 \pm 1.94$ & & $12.96 \pm 1.89$ & 0.769 \\
\hline \multicolumn{2}{|c|}{ Creatinine $(\mu \mathrm{mol} / \mathrm{L})$} & & $1.19 \pm 0.48$ & & $1.11 \pm 0.49$ & 0.529 \\
\hline \multirow[t]{3}{*}{ Child-Pugh } & A & $15(35.7 \%)$ & & $16(32.0 \%)$ & & 0.867 \\
\hline & B & $17(40.5 \%)$ & & $23(46.0 \%)$ & & \\
\hline & $\mathrm{C}$ & $10(23.8 \%)$ & & $11(22.0 \%)$ & & \\
\hline \multirow[t]{3}{*}{ Etiology } & alcohol & $16(38.0 \%)$ & & $17(34.0 \%)$ & & 0.914 \\
\hline & $\mathrm{HBV}$ & $13(31.0 \%)$ & & $17(34.0 \%)$ & & \\
\hline & $\mathrm{HCV}$ & $13(31.0 \%)$ & & $16(32.0 \%)$ & & \\
\hline \multirow[t]{3}{*}{ EV grading } & $\mathrm{F} 1$ & $9(21.4 \%)$ & & $14(28.0 \%)$ & & 0.679 \\
\hline & $\mathrm{F} 2$ & $28(66.7 \%)$ & & $32(64.0 \%)$ & & \\
\hline & F3 & $5(11.9 \%)$ & & $4(8.0 \%)$ & & \\
\hline \multicolumn{2}{|c|}{ Ascites } & $29(69.0 \%)$ & & $35(70.0 \%)$ & & $1.0^{\mathrm{b}}$ \\
\hline
\end{tabular}

Abbreviations: EV, Esophageal varices; HBV, Hepatitis B virus; HCV, Hepatitis C virus; $\mathrm{M} \pm \mathrm{SD}$, Mean \pm standard derivation; N, Number of patients; SBP: Systolic blood pressure.

As disclosed in Table 2, there was no significant difference in the number of EV ligations and the choice of vasoactive medication prescribed between these two groups. Among 92 patients analyzed in this study, 17 cases developed infection during the study period. The frequency of infection was lesser in the antibiotic prophylaxis group than in the control group with statistical significance $(6 \%$ vs. $33.3 \%, \mathrm{P}=0.002)$.

The incidence and type of bacterial infections are shown in Table 3. Bacteremia developed in $2 \%$ and $4.8 \%$ of patients in the antibiotic prophylaxis group and control group respectively. No patients in the antibiotic prophylaxis group developed SBP, but $14.2 \%$ of the control group suffered from this phenomenon. Respiratory tract infection accounted for $2 \%$ and $7.2 \%$ in antibiotic prophylaxis group and control group respectively. UTI developed in $2 \%$ and $7.1 \%$ of patients in antibiotic prophylaxis group and control group respectively. Most bacterial cultures of these infections yielded Gram-negative bacilli (76.9\%). Among 13 micro-organisms found in both groups, 6 bacteria (46\%) were resistant to cefazolin, while the others $(54 \%)$ were sensitive to it. By contrast, all microorganisms were sensitive to quinolone.

As shown in Table 2, the patients in the antibiotic prophylaxis group had less frequent recurrent EV bleeding, or mortality, within 10 days after cessation of previous hemorrhages, but these differences were insignificant ( $6 \%$ vs. $9.5 \%, \mathrm{P}=0.698 ; 5.3 \%$ vs. $7.1 \%, \mathrm{P}=1.0$ ). Among the five dead individuals, four $(80 \%)$ died due to septic shock, and one (20\%) related to active recurrent EV bleeding. The bacteria yielded from dead patients, including Enterobacter cloacae $(\mathrm{n}=2)$, Prevotella melaninogenica $(\mathrm{n}=1)$ and Pseudomonas aeruginosa $(\mathrm{n}=1)$, were all resistant to Cefazolin.

For all the patients involved in this study, mean length of hospital stay was 7.8 days in the control group and 6.6 days in the antibiotic prophylaxis group, and the differences not statistically significant $(\mathrm{P}=1.0)$. No adverse reactions were recorded with antibiotic prophylaxis.

\section{DISCUSSION}

As bacterial infections occur early in cirrhotic patients with variceal bleeding, often because of enteric bacilli, 
Table 2. Vasoactive medication, endoscopic therapy and clinical outcomes of the two groups of patients.

\begin{tabular}{|c|c|c|c|c|c|c|}
\hline \multirow{2}{*}{ Variable } & & \multicolumn{2}{|c|}{ Control Group $\quad(n=42)$} & \multicolumn{2}{|c|}{ Antibiotic Prophylaxis Group $\quad(n=50)$} & \multirow{2}{*}{$P$-value } \\
\hline & & $\mathrm{n} \%$ & $\mathrm{M} \pm \mathrm{SD}$ & $\mathrm{n} \%$ & $\mathrm{M} \pm \mathrm{SD}$ & \\
\hline \multirow[t]{2}{*}{ Medication choose } & Sandostatin & $26(61.9 \%)$ & & $35(70.0 \%)$ & & 0.413 \\
\hline & Glypressin & $16(38.1 \%)$ & & $15(30.0 \%)$ & & \\
\hline \multirow[t]{3}{*}{ Number of EV ligations } & $1-3$ & $8(19.1 \%)$ & & $7(14.0 \%)$ & & 0.763 \\
\hline & $4-6$ & $23(54.8 \%)$ & & $29(58.0 \%)$ & & \\
\hline & $7-9$ & $11(26.1 \%)$ & & $14(28.0 \%)$ & & \\
\hline Infection & & $14(33.3 \%)$ & & $3(6.0 \%)$ & & 0.002 \\
\hline EV Rebleeding & & $4(9.5 \%)$ & & $3(6.0 \%)$ & & 0.698 \\
\hline Mortality & & $3(7.1 \%)$ & & $2(5.3 \%)$ & & 1.0 \\
\hline \multicolumn{2}{|c|}{ Length of hospital stay } & & $7.83 \pm 4.90$ & & $6.62 \pm 2.96$ & 1.0 \\
\hline
\end{tabular}

Abbreviations: EV, Esophageal varices; $\mathrm{M} \pm \mathrm{SD}$, Mean \pm standard derivation; $\mathrm{N}$, Number of patients.

Table 3. Infections and bacterial spectrum of the two groups of patients.

\begin{tabular}{|c|c|c|c|}
\hline & $\begin{array}{l}\text { Control Group }{ }^{a} \\
\quad(n=42)\end{array}$ & $\begin{array}{l}\text { Antibiotic Prophylaxis Group } \\
\qquad(\mathrm{n}=50)\end{array}$ & \multirow{2}{*}{ Bacteria yielded by culture } \\
\hline & $\mathrm{n} \%$ & $\mathrm{n} \%$ & \\
\hline Total infection cases & $14(33.3 \%)$ & $3(6 \%)$ & \\
\hline Bacteremia only & $2(4.8 \%)$ & $1(2 \%)$ & $\begin{array}{l}\text { Bacillus cereus }(\mathrm{n}=1)^{\mathrm{a}}, \\
\text { Klebsiella pneumoniae }(\mathrm{n}=1)^{\mathrm{a}}, \\
\text { Prevotella melaninogenica }(\mathrm{n}=1)^{\mathrm{b}}\end{array}$ \\
\hline SBP & $6(14.2 \%)$ & 0 & $\begin{array}{l}\text { Enterobacter cloacae }(\mathrm{n}=2)^{\mathrm{b}} \\
\text { Klebsiella pneumoniae }(\mathrm{n}=1)^{\mathrm{b}} \\
\text { Escherichia coli }(\mathrm{n}=1)^{\mathrm{a}}\end{array}$ \\
\hline Respiratory tract infction & $2(7.2 \%)$ & $1(2 \%)$ & $\begin{array}{c}\text { Pseudomonas aeruginosa }(\mathrm{n}=1)^{\mathrm{a}} \text {, } \\
\text { Serratia marcescens }(\mathrm{n}=1)^{\mathrm{a}}, \\
\text { Klebsiella pneumoniae }(\mathrm{n}=1)^{\mathrm{b}}\end{array}$ \\
\hline
\end{tabular}

Abbreviations: SBP, Spontaneous bacterial peritonitis; UTI, Urinary tract infection; ${ }^{\text {a }}$ resistant to Cefazolin; ${ }^{\text {b }}$ sensitive to Cefazolon.

we assumed that prophylactic antibiotics in cefazolin treatment could reduce the incidence of infection, as quinolones have been proven to do in previous studies. Studies with prophylactic oral ciprofloxacin reported by $\mathrm{Za}$ charo [16] and Hsieh [11] documented infection reduction from $37.2 \%$ to $10 \%$, and $45 \%$ to $10 \%$, respectively. Prophylactic intravenous ofloxacin adapted by Blaise [10] and Hou [17] showed a lower incidence of infection in the prophylactic group than in the control group, with $20 \%$ vs. $66 \%$ and $3 \%$ vs. $26 \%$ respectively. In a metaanalysis study collated by Bernard [18], it was discovered that the infectious rate for control groups in previous studies ranged from $26 \%$ to $66 \%$, and the ratio of infection decreased up to $20 \%$ after prophylactic antibiotics were adopted. The results of our study with a 10day observation period, similar to previous studies, showed a significant reduction in the incidence of infection from $33.3 \%$ to $6 \%$.

Consistent with former studies [8-10], our study revealed enteric aerobic Gram-negative bacteria are the most common causative organisms in patients with acute gastrointestinal bleeding. Although our study proved the effectiveness of cefazolin in preventing infection in these patients, some resistant-strain bacterial infections appeared not to be protected by cefazolin. By contrast, all bacteria cultured in our study showed sensitivity to quinolones. Therefore, prophylactic quinolones might prevent a greater number of bacterial infections than cefazolin in cirrhotic patients with EV bleeding due to a wider spectrum of bactericidal effectiveness.

The increased incidence of quinolone-resistant bacilli must be weighted. As recent reports show, infection 
caused by quinolone-resistant bacilli markedly increased in patients administered norfloxacin for more than one month [14]. However, bacteria resistance to quinolone were not discovered in our study, meaning quinolone was satisfactory for the clinical conditions at our hospital.

One previous study showed a significant improvement in variceal rebleeding within 6 weeks after acute variceal hemorrhage in patients administered prophylactic antibiotics [17]. This benefit effect of rebleeding was not reflected in our data in a 10-day follow-up period; it may be due to a lower rebleeding rate, shorter observation period in our study and other additional confounding factors altering the relationship of prophylactic antibiotics used and variceal recurrent bleeding.

A previous meta-analysis study by Bernard [18], revealed short-term antibiotic prophylaxis, mainly oral quinolone, significantly increased mean survival rate to 19 days. However, our data suggested similar incidences of mortality within 10 days of admission. In patients who died from septic shock within 10 days of admission, all suffered from cefazolin-resistant bacterial infections. As the majority of mortality occurred after cessation of EV bleeding in cirrhotic patients due to septic shock in our study, prophylactic cefazolin seemed to be ineffective in improving survival.

Similar to previous studies adopting prophylactic quinolone in cirrhotic patients with acute GI bleeding [9-11], our study showed that length of hospital stay was not significantly shorter in patients given prophylactic antibiotics, and this may explain why infections prevented by cefazolin or quinolone were not an independent factor in shortening length of hospital stay.

There were some limitations in our study. Firstly, although about two-thirds of cases of GI bleeding in cirrhotic patients showed variceal hemorrhage, patients analyzed in our study could only reflect conditions of cirrhotic patients with active EV bleeding. Secondly, the varying effectiveness of cefazolin and quinolone in prophylaxis for infection after EV bleeding has not been directly established, and further study comparing the cost-effectiveness of cefazolin and quinolone would be useful. Thirdly, major limitations of this study included the retrospective study design and the lack of randomization. Although the measured baseline characteristics were similar between the antibiotic and control groups, it is possible that there were unmeasured differences between the two groups.

\section{CONCLUSION}

This study provides evidence that prophylactic intravenous cefazolin is effective in preventing bacterial infection for cirrhotic patients with acute EV bleeding, but not of significant benefits for preventing recurrent EV bleeding and mortality within 10 days after initial bleeding.
There is also no significant difference in mean length of hospital stay compared to the control group. We recommend that primary physicians should use prophylactic antibiotics such as quinolone for cirrhotic patients with active EV bleeding according to current guidelines and local bacterial epidemiology.

\section{REFERENCES}

[1] Lee, S.W., Lee, T.Y., Chang, C.S., Ko, C.W., Yeh, H.Z. and Yang, S.S. (2010) Independent factors associated with early outcome in Chinese cirrhotic patients after cessation of initial esophageal variceal hemorrhage. Journal of Clinical Gastroenterology, 44, e123-e127.

[2] Rajkovic, I.A. and Williams, R. (1986) Abnormalities of neutrophil phagocytosis, intracellular killing, and metabolic activity in alcoholic liver cirrhosis and hepatitis. Hepatology, 6, 252-262. doi:10.1002/hep.1840060217

[3] Garcia-Tsao, G., Lee, F.Y., Barden, G.E., Cartun, R. and West, B. (1995) Bacterial translocation to mesenteric lymph nodes is increased in cirrhotic rats with ascites. Gastroenterology, 108, 1835-1841. doi:10.1016/0016-5085(95)90147-7

[4] Bleichner, G., Boulanger, R., Squara, P., Sollet, J.P. and Parent, A. (1986) Frequency of infections in cirrhotic patients presenting with acute gastrointestinal haemorrhage. British Journal of Surgery, 73, 724-726. doi:10.1002/bjs. 1800730916

[5] Goulis, J., Armonis, A., Patch, D., Sabin, C., Greenslade, L. and Burroughs, A.K. (1998) Bacterial infection is independently associated with failure to control bleeding in cirrhotic patients with gastrointestinal hemorrhage. Hepatology, 27, 1207-1212. doi:10.1002/hep.510270504

[6] Bernard, B., Cadranel, J.F., Valla, D., Sabin, C., Greenslade, L. and Burroughs, A.K. (1995) Prognostic significance of bacterial infection in bleeding cirrhotic patients: a prospective study. Gastroenterology, 108, 1828-1034. doi:10.1016/0016-5085(95)90146-9

[7] Lee, S.W., Lee, T.Y. and Chang, C.S. (2009) Independent factors associated with recurrent bleeding in cirrhotic patients with esophageal variceal hemorrhage. Digestive Diseases and Sciences, 54, 1128-1134. doi:10.1007/s10620-008-0454-0

[8] Rimola, A., Bory, F., Teres, J., Perez-Ayuso, R.M., Arroyo, V. and Rodes, J. (1985) Oral, nonabsorbable antibiotics prevent infection in cirrhotics with gastrointestinal hemorrhage. Hepatology, 5, 463-467. doi:10.1002/hep. 1840050320

[9] Soriano, G., Guarner, C., Tomas, A., Villanueva, C., Torras, X., Gonzalez, D., et al. (1992) Norfloxacin prevents bacterial infection in cirrhotics with gastrointestinal hemorrhage. Gastroenterology, 103, 1267-1272.

[10] Blaise, M., Pateron, D., Trinchet, J.C., Levacher, S., Beaugrand, M. and Pourriat, J.L. (1994) Systemic antibiotic therapy prevents bacterial infections in cirrhotic patients with gastrointestinal hemorrhage. Hepatology, 20, 34-38. doi:10.1002/hep.1840200107

[11] Hsieh, W.J., Lin, H.C., Hwang, S.J., Hou, M.C., Lee, F.Y. 
and Chang, F.Y. (1998) The effect of ciprofloxacin in the prevention of bacterial infection in patients with cirrhosis after upper gastrointestinal bleeding. American Journal of Gastroenterology, 93, 962-966. doi:10.1111/j.1572-0241.1998.00288.x

[12] Fernandez, J., Ruiz del Arbol, L., Gomez, C., Durandez, R., Serradilla, R., Guarner, C., et al. (2006) Norfloxacin vs ceftriaxone in the prophylaxis of infections in patients with advanced cirrhosis and hemorrhage. Gastroenterology, 131, 1049-1056. doi:10.1053/j.gastro.2006.07.010

[13] Garcia-Tsao, G., Sanyal, A.J., Grace, N.D. and Carey, W. (2007) Prevention and management of gastroesophageal varices and variceal hemorrhage in cirrhosis. Hepatology, 46, 922-993. doi:10.1002/hep.21907

[14] Ortiz, J., Vila, M.C., Soriano, G., Minana, J., Gana, J., Mirelis, B., et al. (1999) Infections caused by Escherichia coli resistant to norfloxacin in hospitalized cirrhotic patients. Hepatology, 29, 1064-1069. doi:10.1002/hep.510290406

[15] Castellote, J., Xiol, J., Rota Roca, R. and Fernandez Es- parrach, G. (1994) Spontaneous bacterial peritonitis and empyema by Escherichia coli resistant to norfloxacin in a patient on selective intestinal decontamination with norfloxacin. Journal of Hepatology, 20, 436. doi:10.1016/S0168-8278(94)80022-7

[16] Zacharof, A.K., Petrogiannopoulos, C., Flevaris, C. and Deliousis, A. (1997) Ciprofloxacin prevents bacterial infection in cirrhotics with gastrointestinal hemorrhage. Journal of Hepatology, 26, 101.

[17] Hou, M.C., Lin, H.C., Liu, T.T., Kuo, B.I., Lee, F.Y., Chang, F.Y. and Lee, S.D. (2004) Antibiotic prophylaxis after endoscopic therapy prevents rebleeding in acute variceal hemorrhage: A randomized trial. Hepatology, 39, 746-753. doi:10.1002/hep.20126

[18] Bernard, B., Grange, J.D., Khac, E.N., Amiot, X., Opolon, P. and Poynard, T. (1999) Antibiotic prophylaxis for the prevention of bacterial infections in cirrhotic patients with gastrointestinal bleeding: A meta-analysis. Hepatology, 29, 1655-1661. doi:10.1002/hep.510290608 